\title{
Guest-editorial
}

\section{Computational aspects of electric polarizability calculations: Atoms, molecules and clusters}

\author{
George Maroulis \\ Department of Chemistry, University of Patras, Patras, Greece \\ E-mail:maroulis@upatras.gr
}

The theory of electric (hyper)polarizability constitutes the cornerstone of the rational approach to a wide spectrum of phenomena. Such phenomena range from nonlinear optics and phenomena induced by intermolecular interactions to electron scattering. It is also worth emphasizing that polarizability is now routinely associated with important characteristics of electronic structure as hardness/softness, acidity/basicity or properties as the ionization potential. The prediction of reliable values for electric polarizability by rigorous quantum chemical methods has made significant contributions and added new vigor to intensively active fields as the search for new nonlinear optical materials, collision- and interaction-induced spectroscopy, molecular simulation and modeling of fundamental processes and the determination of molecular structures and properties of weakly bonded van der Waals molecules. In addition, electric polarizability is now of some importance to modern pharmacology and is extensively used as a molecular descriptor in QSAR studies. Some of the above fields possess considerable potential for advanced technological applications.

This special issue brings together a representative selection of papers focusing on computational aspects of theoretical determinations of electric polarizability. Our aim is to show the color and content of current investigations by a large number of research groups. We have classified the papers into six categories, divided over two journal issues. The details are noted below. This classification is, as we readily admit, by no means unique. Nevertheless, it brings forth the amazing wealth of significant results made available in recent years.

Professor George Maroulis Guest-editor, November 2004 